\title{
Analysis of the ventilation systems in the Dartford tunnels using a multi- scale modelling approach
}

\author{
F. Colella, G. Rein, R. Carvel, P. Reszka, J.L. Torero \\ University of Edinburgh, BRE Centre for Fire Safety Engineering, UK
}

\begin{abstract}
The capabilities of the ventilation systems in the two road tunnels at Dartford (UK) are analysed using a multi-scale modelling approach. Both tunnels have complex semi-transverse ventilation systems with jet fans to control longitudinal flow. The construction and ventilation systems in the tunnels are described and the current emergency ventilation strategies are presented. The analysis includes a coupling of a 1D network model with 3D components, representing the operational jet fans, built using computational fluid dynamics. The jet fans were experimentally characterized on-site and the findings were compared to the model predictions. The predicted ventilation flows for each of the emergency ventilation strategies are presented and discussed. In cold-flow conditions, ventilation velocities significantly above $3 \mathrm{~m} / \mathrm{s}$ can be generated throughout the tunnels. However, it is observed that $1 / 3$ of the flow generated in the East tunnel is diverted from the tunnel up the extract shafts. The model was used to simulate various reduced fan combinations and thus the level of redundancy in each of the systems has been estimated. It is found that an acceptable level of ventilation may be produced in the West tunnel, even if several pairs of jet fans are disabled. In the East tunnel there is less redundancy, but an acceptable level of ventilation control can be maintained with one or two jet fans disabled.
\end{abstract}

\section{Introduction}

The Dartford tunnels are two twin-lane, uni-directional road tunnels under the River Thames, crossing from Dartford at the south (Kent) side of the river to Thurrock at the north (Essex)

side, about 15 miles east of London in the UK. Both tunnels have complex ventilation systems consisting of a semi-transverse system together with additional jet fans to control longitudinal flow.

The control systems in the tunnels are currently being upgraded and a new analysis is required, specifically to identify how many components of the ventilation system can be disabled for refurbishment whilst still maintaining an acceptable level of safety throughout the tunnel.

Previous (unpublished) analyses of the ventilation systems utilised detailed computational fluid dynamics (CFD) models of the tunnels to predict the capabilities of the fully operational system under each of the four emergency ventilation strategies currently used in the tunnels. Given the size of each of the computational domains and the necessary mesh detail, the computational time for each of the simulations was several days. As the intent of this study was to study the capabilities of the ventilation systems across the whole range of possible reduced configurations, it was simply not practical to utilise a full CFD approach due to the number of simulations required.

The development of new multi-scale modelling techniques (Colella et al., 2009) enables simulations to be run more efficiently and thus allows the analysis of many more ventilation combinations than was possible in the previous study. This is because multiscale modelling does not require the construction a full-length CFD model of the tunnel, only small portions of it, and can provide valid ventilation results for any position along the tunnel. Thus it has become possible to compare various ventilation strategies and identify the minimum and optimum fan combinations to use for various scenarios. The multiscale model is a new technique which has not yet been the subject of a rigorous validation study. It is possible to use such a model to predict ventilation capabilities in complex tunnel configurations containing fans, obstacles and fires (Colella et al., 2010). However, in this paper the model is validated only against cold-flow ventilation measurements carried out in the Dartford tunnels. Obviously, it has not been possible to carry out fire experiments in the tunnels, so the ability of the model to simulate the influence of a fire has not yet been validated. Thus, this paper will concern only the validated cold-flow simulations.

\section{Description of the tunnels}

The tunnels are approximately $1.5 \mathrm{~km}$ long and each tunnel carries uni-directional traffic in two lanes. Generally, both tunnels carry northbound traffic only, while southbound traffic uses the four lane Queen Elizabeth II bridge, which lies slightly to the east of the tunnels. In instances of extreme 


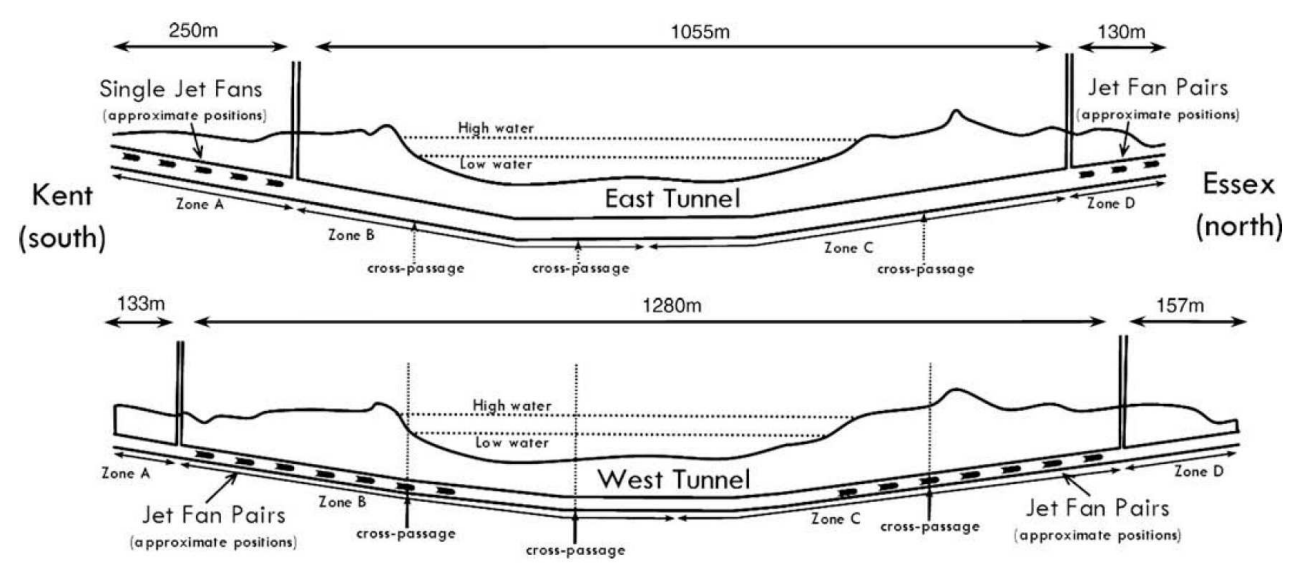

Fig. 1. Diagram of the East and West tunnels showing the relative positions of the jet fans and extract shafts. (Drawn approximately to scale but with vertical distances five times larger.)

weather, the bridge may be closed and the traffic direction in the East tunnel may be switched to southbound.

Fig. 1 shows the general layout of the tunnels. The West tunnel (approximately $8.6 \mathrm{~m}$ internal diameter) was opened to traffic in 1963 and the East tunnel (approximately $9.5 \mathrm{~m}$ internal diameter) in 1980. The West tunnel is constructed of a cast iron segmental lining, which has been infilled with concrete. The East tunnel is constructed of three different types of primary lining material: the central $600 \mathrm{~m}$ of the tunnel are constructed of pre-cast concrete segments with steel face plates, on either side of the central section there is a portion of the tunnel (170 mlong at the north end and 100 mlong at the south) constructed of cast iron segments, the remainder of the tunnel $(200 \mathrm{~m}$ at the north and $355 \mathrm{~m}$ at the south) was constructed of cast in situ concrete using a cut and cover technique.

In both tunnels the semi-transverse ventilation system has two shafts with axial extraction fans located at relatively short distance from each of the tunnel portals. In both tunnels the semi-transverse system supplies fresh air into the tunnel through grills along the side of the roadway between the extract shafts. The fresh air is pumped into the invert under the roadway by means of two axial fans, one at the Kent end and one at Essex. There are no transverse supply grills between the portals and the shafts. In the West tunnel, there are 14 pairs of uni-directional jet fans, installed in niches, located between the extract shafts. The fans in the East tunnel are more powerful and are not installed in niches. There are five individual reversible jet fans between the southern portal and the southern extract shaft and three pairs of reversible jet fans between the northern shaft and the northern portal. The jet fan spacing is around $50 \mathrm{~m}$ in both the tunnels. The layout of the tunnels and the position of the jet fans is shown in Fig. 1.

In the event of a fire, the emergency strategy currently implemented in the Dartford tunnels assumes that all vehicles ahead of the incident will be able to safely exit the outgoing portal, while a queue of traffic builds up behind the incident. Thus, the ventilation is configured in such a way as to blow any smoke away from the queuing traffic. To allow for a flexible emergency response, four different ventilation strategies are used, depending on the location within the tunnel where the fire occurs:

- If the incident occurs between the Kent portal and the southern extract shaft (hereafter referred to as 'Zone $A$ '), the ventilation strategy utilises the activation of all jet fans (blowing south to north) and both extract fans, but sets both supply fans off.

- If the incident occurs between the southern extract shaft and the mid point of the tunnel ('Zone B'), the ventilation strategy utilises the activation of all jet fans (blowing south to north) and the northern extract fan, but sets both supply fans and the southern extract fan off.

- If the incident occurs between the mid point of the tunnel and the northern extract shaft ('Zone C'), the ventilation strategy utilises the activation of all jet fans (blowing south to north), the southern supply fan and the northern extract fan, but sets the northern supply fan and the southern extract fan off.

- If the incident occurs between the northern extract shaft and the Essex portal ('Zone D'), the ventilation strategy utilises the activation of all jet fans (blowing south to north) and both supply fans, but sets both extract fans off.

An innovative multi-scale model of the ventilation has been developed and used to quantify the 


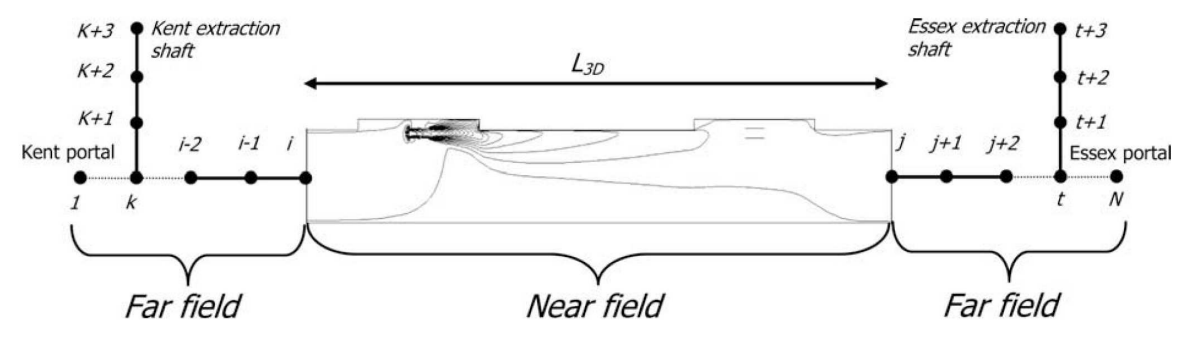

Fig. 2. Schematic of multi-scale coupling between mono-dimensional and CFD models.

actual ventilation system capabilities. In both the tunnels the multi-scale model has been used to characterize the jet fan discharge cone and to explore several other ventilation strategies. A comprehensive study has been conducted to assess the capabilities of reduced fan combinations, to demonstrate the level of redundancy in the systems and, hence, to calculate the number of fans that may be safely disabled for maintenance or refurbishment purposes.

\section{Multi-scale model}

The study of ventilation flows in tunnels (Colella et al., 2009) provides evidence that, in the vicinity of operating jet fans, the flow field has a complex 3D behaviour with large transversal and longitudinal velocity gradients. The flow in these regions cannot be accurately represented using a 1D model and needs to be calculated using a 3D modelling tool such as CFD. These regions are hereafter referred to as the near field. However, it has been demonstrated for cold-flow scenarios (Colella et al., 2009) that some distance away from this region, the velocity gradients in the transversal direction tend to disappear and the flow becomes approximately 1D. In this region, the transversal components of the flow velocity can be up to two orders of magnitude smaller than the longitudinal component. These regions are hereafter named as the far field. The use of CFD models to simulate the fluid behaviour in the far field leads to big increases in the computational requirements but very small improvements in the accuracy of the results.

The adoption of multi-scale models represents a way to avoid the large computational cost of the full CFD and the inaccuracies of simplistic assumptions in 1D models. A multi-scale method uses different levels of detail when describing the fluid dynamic behaviour of different tunnel regions. The behaviour of far field regions is modelled using a $1 \mathrm{D}$ 'network' model and the near field using a CFD model. The network and the CFD models exchange information at the 1D-3D interfaces and thus are able to run in parallel. A schematic of the coupling is presented in Fig. 2.

The general methodology of the 1D network model of the far field has been presented elsewhere (Colella et al., 2009; Ferro et al., 1991) and only a brief overview is given here. The first step is to generate a network of elements representing the tunnel layout. The longitudinal domain is discretised in oriented elements called branches, interconnected by nodes. The nodes are volumeless elements connecting different branches. Mass conservation equations in each node and momentum conservation equation written following the generalized Bernoulli formulation are solved by the model (Ferro et al., 1991; Jacques, 1991; Riess et al., 2000; Cheng et al., 2001; Jang and Chen, 2002). The model is able to account for the pressure loss due to blockages (minor losses) and wall friction (major losses) computed using the Colebrook formula (Fox and McDonald, 1995).

The pressure gain by a fan inside a branch, also called the fan characteristic curve, is a function of the flow velocity through the branch and can be represented in general by a polynomial of second order (Ferro et al., 1991; Jacques, 1991; Riess et al., 2000; Cheng et al., 2001; Jang and Chen, 2002). In most cases, the fan characteristic curve is unknown or insufficiently quantified because fan behaviour is strongly coupled with the surrounding tunnel components and is thus dependent on the specific installation configuration (Jang and Chen, 2002). The jet fan thrust curve provided by a manufacturer only applies to the jet fan in isolation and not once installed in a particular tunnel geometry. Usually, in situ measurements or further CFD analysis are required in order to accurately quantify the fan characteristic curve.

The computation of the near field has been carried out using the finite volume CFD tool FLUENT (Fluent Inc., 2005). In this particular application a segregated solver has been used to solve the Navier-Stokes equations. In order to take care of the complex turbulent phenomena within the domain, two more additional equations

for the turbulent quantities need to be solved. The ke turbulence model has been largely validated by the scientific community is still the most practical and least time consuming approach. The standard k-e model is not valid for regions close to the walls which are characterized by a low Reynolds number (Versteeg and Malalasekera, 2007). For these regions, a standard wall function approach has been used instead.

The wall function approach is valid as long as the first mesh point is located within the logarithmic region of the boundary layer (i.e., yp + ranges between 30 and 300). 


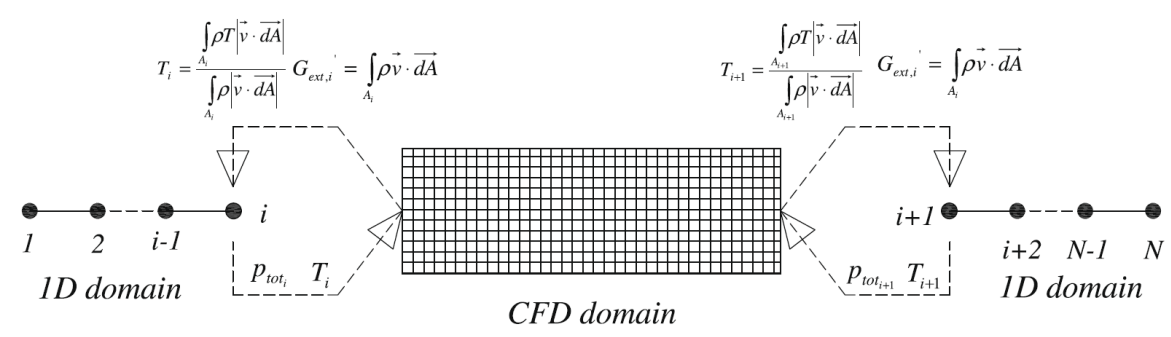

Fig. 3. Boundary conditions exchange for the direct coupling of the 1D and CFD models.

A posteriori calculations have been performed in all CFD cases presented here to confirm that this condition is reached.

The CFD model also requires a representation of the jet fans. Previous works on the subject (Karki and Patankar, 2000; Vega et al., 2008) simulated the jet fans as a combination of sources and sinks. This kind of approach has not been used in this paper in order to avoid discrepancies in the conservation of energy, mass, species or momentum that can be generated by uncoupling discharge and intake surfaces. The proposed methodology simulates the jet fans as fluid regions delimitated by cylindrical solid walls and containing a cross-sectional surface where a constant, positive pressure jump is imposed.

The 3D tunnel domain has been discretized using a quasi-structured mesh with refinements introduced in the proximity of jet fans and walls.

The mesh density has been chosen on the basis of the grid independence study conducted in Colella et al. (2009). In that work it has been demonstrated that a grid independent solution was reached for mesh densities above 4000 cells $/ \mathrm{m}$ of tunnel length. The evaluation of the fluid dynamic quantities at the finite volume interface used a second order upwind scheme for the convective and pressure terms (Fluent Inc., 2005). The solution is assumed to be converged when the scaled residuals were lower than $10^{-5}$, except for the energy equation where $10^{-7}$ is used.

The 1D and CFD domains exchange information at the interfaces, between nodes $i$ and $i+1$ of Fig. 3 . The coupling can be conducted in two different ways; direct and indirect coupling.

For most ventilation studies, bulk flow velocities or average temperature values in steady state or quasi steady state conditions are needed. In this case an indirect coupling method can be adopted and $1 \mathrm{D}$ and $3 \mathrm{D}$ simulations may be run separately. After identifying the near field(s), a series of CFD runs are conducted for the near field domain(s), for a range of uniform boundary conditions at the interfaces. In this manner, the CFD results are arranged in terms of bulk flow velocities as a function of the total pressure differences across the near field domain(s) allowing the definition of the characteristic curves. This curve represents the coupling of the active element of interest (shaft, jet fan, blockage, fire, etc.) with the surrounding tunnel gallery.
These curves calculated by the CFD model are built into the 1D network model, coupling them with the rest of the tunnel. Obviously it is crucial that the 3D near field domain must be sized appropriately, such that the boundary interfaces are located in regions where the flow is fully developed and approximately $1 \mathrm{D}$ in nature.

Implementing the model in this indirect way allows the prediction of steady state ventilation behaviour for numerous scenarios to be carried out by changing only the parameters of the network model, without having to re-run any 3D models. For most tunnel ventilation analyses, this represents a considerable reduction in the time taken and the computational resources used.

Conversely, using direct coupling, the 1D model is embedded within the CFD model and these are run iteratively together. The solution of the multi-scale model requires a constant exchange of information during the solution procedure. Direct coupling is required if details of the 3D flow field directly feedback to the 1D bulk flow, for example simulating the transient response of jet fan activation or rapid fire growth. The direct coupling algorithm has to perform the following operations at each iteration step:

1. Provide the CFD model with the pressure and temperature boundary conditions at the interfaces $i$ and $i+1$ calculated by the $1 D$ model.

2. Perform one iteration of the CFD model using a segregate solver.

3. Integrate the air velocities at the interfaces $i$ and $i+1$ to calculate the global mass flow rate and fix them as boundary for the $1 \mathrm{D}$ model (see Fig. 3).

4. Average the temperature at the interfaces $i$ and $i+1$ to provide the boundary conditions for the 1D model (see Fig. 3).

\section{Solve the 1D model.}

6. Repeat back to step 1 until convergence is reached. 
F. Colella, G. Rein, R. Carvel, P. Reszka, J.L. Torero, (2010) Analysis of the ventilation systems in the Dartford tunnels using a multi-scale modelling approach, Tunnelling and Underground Space Technology 25, pp. 423-432. Open access version at http://www.era.lib.ed.ac.uk/handle/1842/1152

\section{Proceed to the following time step (for transient calculations).}

Multi-scale modelling techniques allow a significant reduction of the computational time (up to two orders of magnitude). This paper presents an application of this novel technique to conduct a comprehensive analysis of the ventilation system in the Dartford tunnels. Both indirect and direct coupling strategies have been used: the first technique to characterize the ventilation system under various reduced capacity scenarios and the second one to model the jet fan discharge cones. To the best knowledge of the authors, this is the first time that this technique has been applied and validated against experimental data from real operating tunnels.

\section{Characterization of the jet fan discharge cone}

An assessment of the performance of the tunnel ventilation system was undertaken requiring a detailed study of the flow field around the jet fans.

In particular, the analysis of the discharge cone from a single operating jet fan (or jet fan pair) was required for both tunnels to understand how their ability in producing thrust depends on the particular installation details in Dartford.

For this purpose an experimental campaign was performed and some modelling attempts were made in order to reproduce the flow field around the jet fans. The measurements were taken with only the 5th jet fan pair operating in the West tunnel and only the 3rd jet single jet fan operating in the East tunnel (see Fig. 1).

Since the local details of the flow field are required, a multiscale model with direct coupling was used. For this analysis, the tunnel domain was split into a near field region where a CFD model was used and a far field region modelled using a 1D approach. A visual example of the model arrangement for this specific application is presented in Fig. 2. The effects of the air supply network and the other (non operational) jet fans were deemed to be negligible, thus have been omitted from the model to keep the analysis simple. The length of the CFD domain (L3D in Fig. 2) has been chosen on the basis of a boundary independence study (Colella et al., 2009). The analysis confirmed that the accuracy of the multiscale model is improved up to around $1 \%$ by using $300 \mathrm{~m}$ as length of the near field which in this case corresponds to around $20 \%$ of the tunnel

length.

The multi-scale model results were compared to experimental velocities measured using a portable rotating vane anemometer (calibrated Kimo AMI301 $70 \mathrm{~mm}$ diameter). This instrument provides measurements in the range $0.3-35 \mathrm{~m} / \mathrm{s}$ with an accuracy of $2 \% \pm 0.1 \mathrm{~m} / \mathrm{s}$. This anemometer provides low errors for flow-misalignment angles up to $24^{\circ}$.

The longitudinal air velocities have been recorded at various points across the cross-section, at six different locations, at $20 \mathrm{~m}$ intervals, starting $20 \mathrm{~m}$ downstream from the jet fan discharge surface. In each section, nine velocity points have been considered. Fig. $4 a$ and $b$ shows the spatial location of the points where the velocities were measured. The total experimental error of the measurements has been estimated to be $\pm 14.5 \%$. This value has been included as error bars in Fig. 5 .

The comparison between predicted and experimental velocities measured in the West tunnel is presented in Fig. 5. The blue continuous

line represents the velocity profiles calculated in the middle of the tunnel cross-sections (profile 1 in Fig. 4a) while the red dashed ones represent the velocity profiles calculated along the vertical lines corresponding to the profiles 2 in Fig. $4 a$.

The measured velocity values represented in Fig. 5 are numbered from 1 to 9 following the same pattern as presented in Fig. 4a. The comparison is quite encouraging as in almost all the measurement sections there is a good agreement between experimental and numerical data.

All the CFD tests done during the development of the model have demonstrated that the niches where the jet fans are located have a significant effect on the longitudinal development of the flow and their capability of producing thrust. The poorest agreement between the model and the experimental data was found in the section $60 \mathrm{~m}$ downstream from the fan pair where the maximum relative deviation did not exceed $30 \%$; this peculiarity is most likely due to the presence of obstacles located on the tunnel ceiling (other fans and lighting devices) which are not included in the computational domain but will influence the discharge cone characteristics.

A similar degree of accuracy is obtained when comparing predicted and measured velocity profiles in the East tunnel (see Fig. 6). In this case there was only one operating jet fan and therefore the velocity profile is not symmetrical across the tunnel longitudinal plane, unlike in the West tunnel.

The blue continuous line represents the velocity profiles calculated in the middle of the tunnel crosssections (profile 2 in Fig. 4b). The red velocity profile with finer dashing represents the velocity calculated on the vertical line indicated as profile 1 in Fig. $4 \mathrm{~b}$. The green velocity profile with coarser dashing represents the velocity calculated on the vertical line indicated as profile 3 in Fig. $4 \mathrm{~b}$.

The measured velocity values represented in Fig. 6, are numbered from 1 to 9 following the same pattern as presented in Fig. 4b. Also in this case the comparison is quite encouraging as in almost all the measurement sections there is a good agreement between experimental and numerical data. The poorest agreement between experimental and numerical data has been encountered in the section 
F. Colella, G. Rein, R. Carvel, P. Reszka, J.L. Torero, (2010) Analysis of the ventilation systems in the Dartford tunnels using a multi-scale modelling approach, Tunnelling and Underground Space Technology 25, pp. 423-432. Open access version at http://www.era.lib.ed.ac.uk/handle/1842/1152
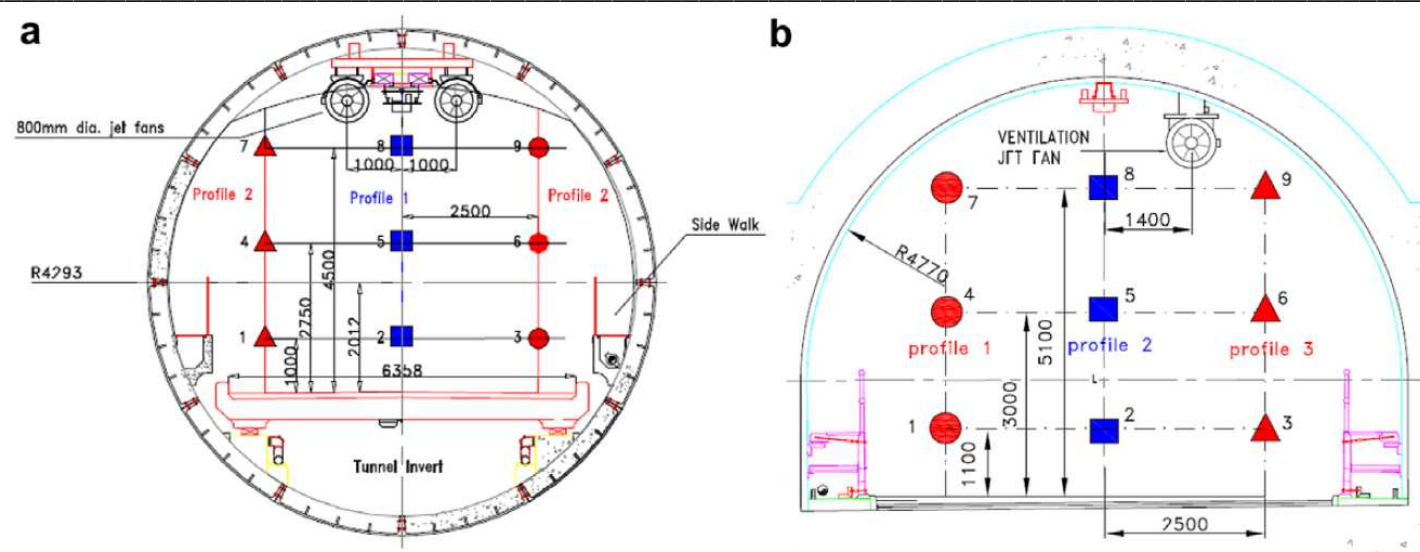

Fig. 4. Layout and general dimensions of the tunnel cross-sections (West tunnel to the left; East tunnel to the right) including the points 1-9 where the air velocities were measured (dimensions are in $\mathrm{mm}$ ).
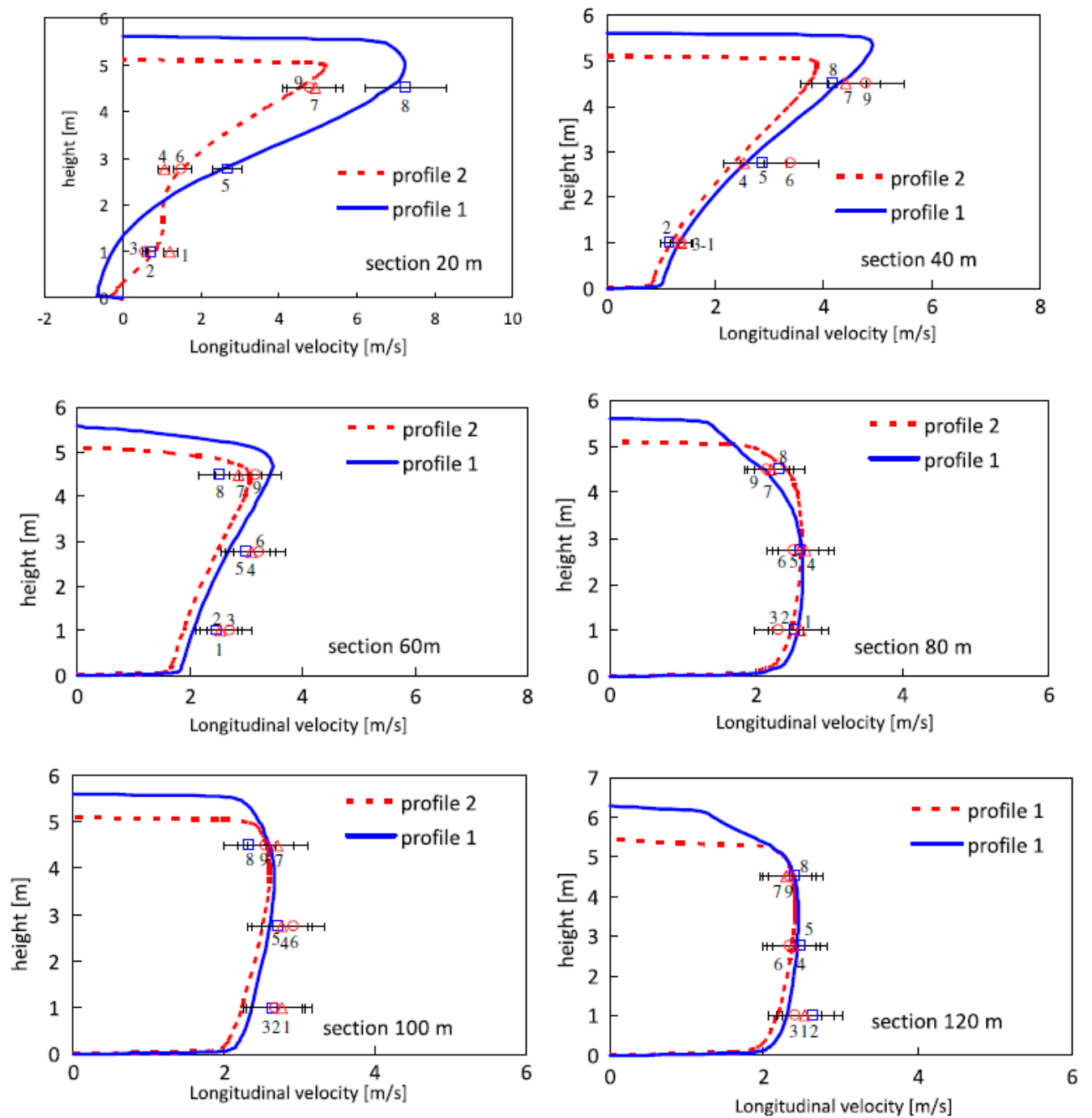

Fig. 5. Comparison of horizontal velocities between predictions (lines) and experimental measurements (symbols) in the West tunnel. The two profiles and the numbers refer to locations in the tunnel section described in Fig. 4a. 

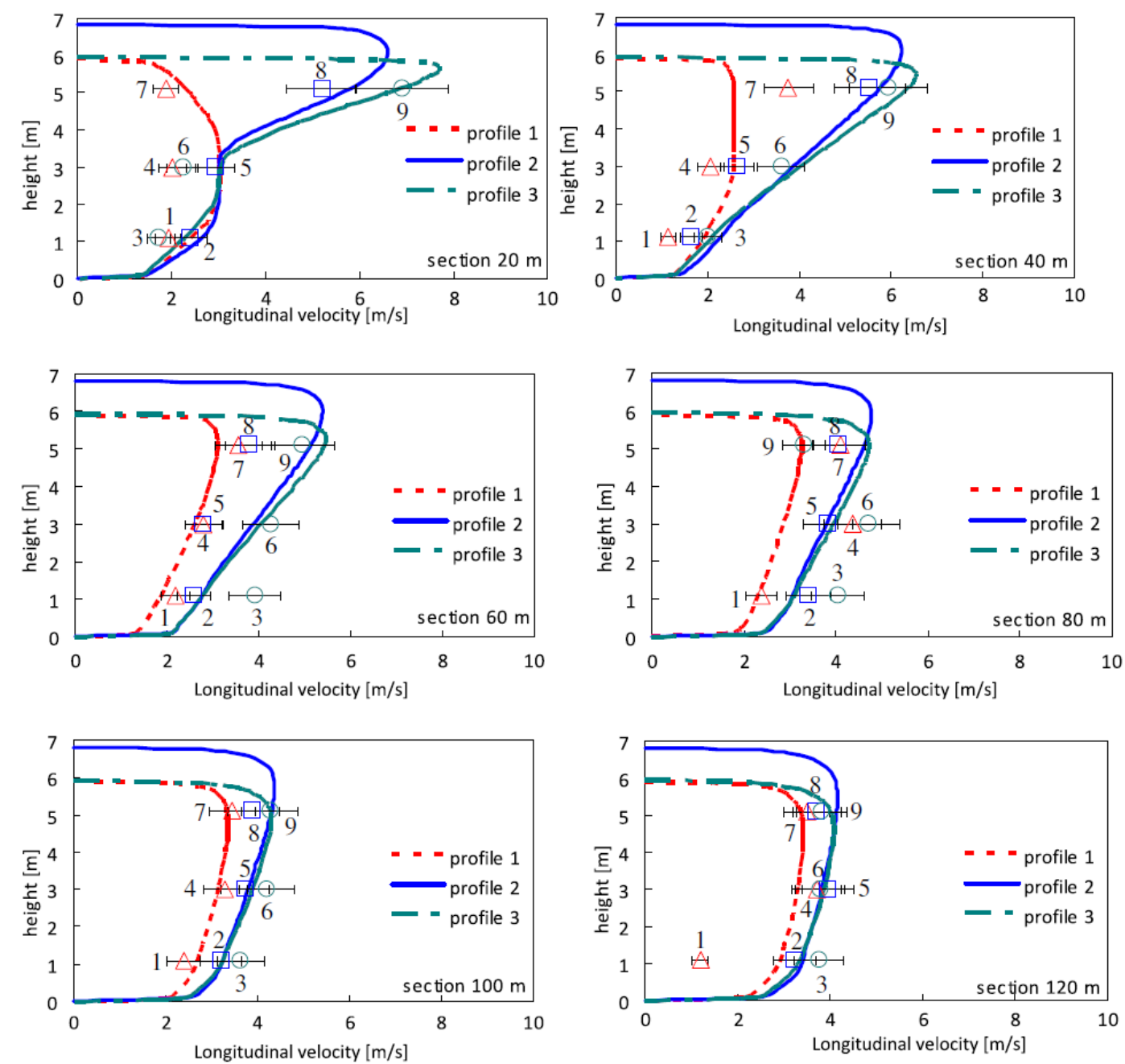

Fig. 6. Comparison of horizontal velocities between predictions (lines) and experimental measurements (symbols) in the East tunnel. The three profiles and the numbers refer to locations in the tunnel section described in Fig. $4 \mathrm{~b}$.

$80 \mathrm{~m}$ downstream from the fan where the maximum relative deviation did not exceed $20 \%$. This is most likely due to obstacles located in the specific region of the tunnel (luminaires or other jet fans) or to a sudden change in the meteorological conditions given the strong external winds recorded during the measurement campaign.

The analysis of the jet fan discharge cone confirmed that the flow is approximately one-dimensional in nature beyond $80 \mathrm{~m}$ downstream of the fan outlet in the case of the West tunnel. In the East tunnel the discharge cone is slightly longer at $100 \mathrm{~m}$. This is because the jet fans installed within the East tunnel are more powerful than in the West, and not installed in niches on the ceiling, as they are in the West tunnel.

When more than one jet fan pair is operating, the near field must include all operating devices within the module length. Alternatively and computationally more efficiently, the jet fan series can be modelled by including a single representative CFD module which operates in a periodic behaviour. The periodic flow boundary condition was shown in Colella et al. (2009) to provide good results.

\section{Characterization of the ventilation system}

The assessment of the ventilation system performance required a comprehensive study of the ventilation strategies within the tunnels. In particular, the study aims to understand the consequences on the tunnel flow of making changes to the fan configurations. This kind of analysis does not require detailed flow field data but only bulk flow velocities within the tunnel domain.

The first modelling choice to address this problem was a purely 1D model. For this particular application, the main difficulty encountered when using the 1D approach was related to the assessment of the jet fan thrust and their losses induced by their peculiar installation locations (i.e. in niches in the West tunnel). In fact, it is well known 

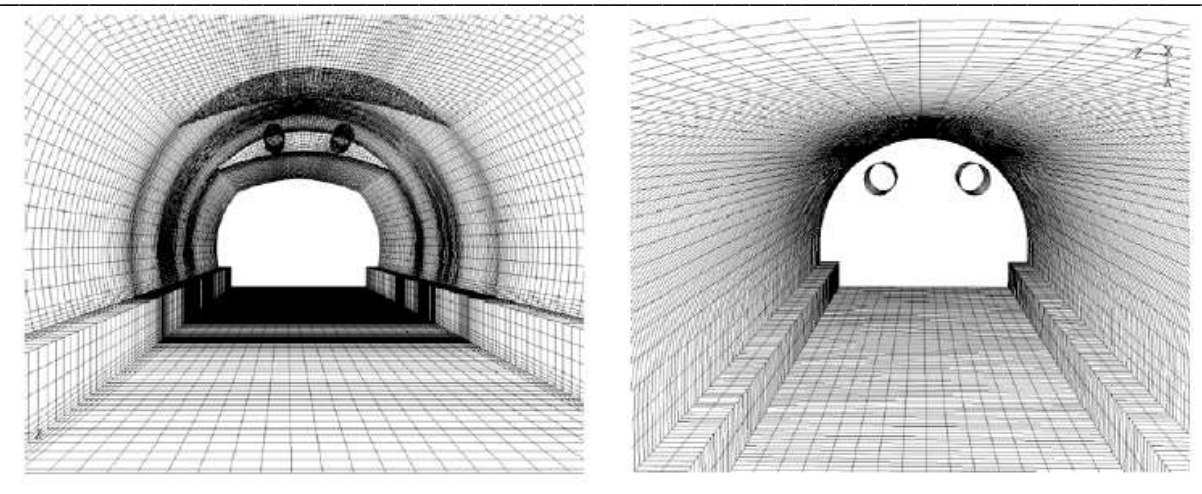

Fig. 7. Computational mesh for the CFD module around the jet fans in the West (right) and East (left) tunnels. (Note: the West tunnel's jet fans are installed in niches on the ceiling, in the East tunnel they are not.)

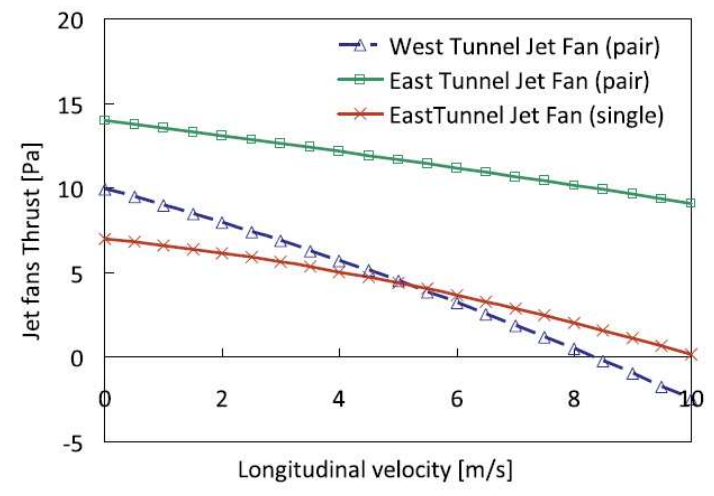

Fig. 8. CFD calculated jet fan thrust vs. tunnel average velocity for the Dartford tunnels. (Curves computed for CFD module length of $50 \mathrm{~m}$ ).

that the pressure rise produced by the jet fans is strictly dependent on the specific surrounding environment (Jang and Chen, 2002). Therefore the prediction capability of a $1 \mathrm{D}$ model mainly relies on calibration constant to be defined arbitrarily or on the basis of literature data. Furthermore some empirical correlations to estimate the thrust from jet fan pairs were adopted (Ferro et al., 1991; Jacques, 1991; Riess et al., 2000; Cheng et al., 2001; Jang and Chen, 2002) but, in several cases, they overpredicted the actual capabilities of the ventilation system.

In order to overcome this problem, a multi-scale modelling approach with indirect coupling was used. This kind of approach is based on the determination of the near field characteristic curves which are then introduced as input in a simple 1D model improving is prediction capabilities.

Two schematics of the CFD meshes used for the purpose are presented in Fig. 7. Fig. 8 shows the characteristic curve of the West and East jet fans presented in terms of bulk flow velocity vs. total pressure difference. The curves describe the capability the jet fans to produce thrust and its dependence on the mass flow velocity. The characteristic curve of the fan region where more than one fan is operating is calculated by using the methodology presented in Colella et al. (2009). The approach is based on the hypothesis that the series of operating jet fans produces a flow field characterized by a periodic pattern. The upstream

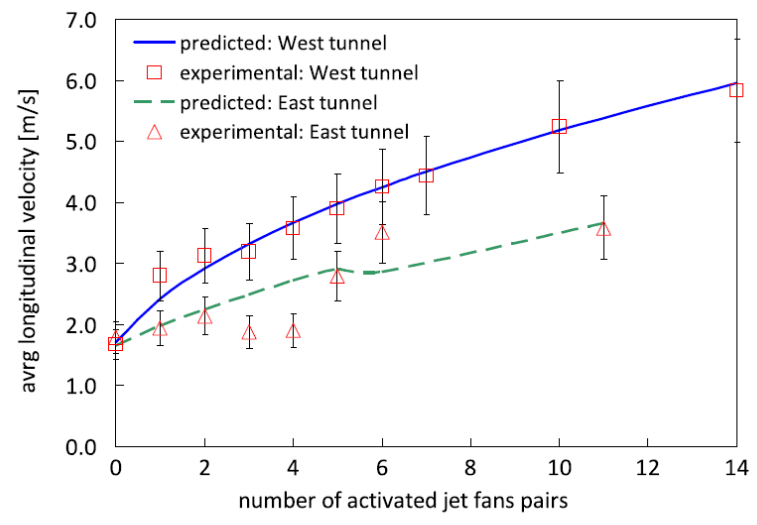

Fig. 9. Comparison between experimental data and modelling predictions.

and downstream boundaries have been defined as periodic surfaces. The hypothesis is confirmed to be valid using CFD simulation of a train of operating jet fans (Colella et al., 2009).

The results of this CFD study for the near field are then coupled to the 1D model for the rest of the tunnel. Specifically, the computed curves are used as the characteristics of any branch of the 1D model containing jet fans avoiding the uncertainties related to calibration constants.

It is worth to highlight that the assumed flow periodicity lasts as long as the supply fans within the tunnel are off. The introduction of fresh air from the transverse supply will slightly modify the velocity patterns within the tunnel and the mass flow rate will not be constant along the longitudinal direction. However, these effects are small as the amount of fresh air introduced in the fan region is negligible compared to the large mass flow rate through the main gallery. Thus, in this case, the computed characteristic curve provides a good approximation which can be successfully applied.

The multi-scale model with indirect coupling has been validated using bulk flow data measured in the central section of the tunnels under a range of fan combinations. The comparison between predictions and recorded bulk velocities is presented in Fig. 9 as a function of the number of operating jet fans. The agreement between the experimental data and the predictions is excellent, demonstrating accurate prediction capabilities. Some discrepancies can be 
F. Colella, G. Rein, R. Carvel, P. Reszka, J.L. Torero, (2010) Analysis of the ventilation systems in the Dartford tunnels using a multi-scale modelling approach, Tunnelling and Underground Space Technology 25, pp. 423-432. Open access version at http://www.era.lib.ed.ac.uk/handle/1842/1152

\begin{tabular}{|c|c|c|c|c|c|c|c|c|c|}
\hline \multirow[t]{2}{*}{ Ventilation strategy } & & \multirow{2}{*}{$\begin{array}{l}\text { All jet } \\
\text { fans }\end{array}$} & \multirow{2}{*}{$\begin{array}{l}\text { Kent axial } \\
\text { extract fan }\end{array}$} & \multirow{2}{*}{$\begin{array}{l}\text { Essex axial } \\
\text { extract fan }\end{array}$} & \multirow{2}{*}{$\begin{array}{l}\text { Kent axial } \\
\text { supply fan }\end{array}$} & \multirow{2}{*}{$\begin{array}{l}\text { Essex axial } \\
\text { supply fan }\end{array}$} & \multicolumn{3}{|c|}{ Ventilation velocities $(\mathrm{m} / \mathrm{s})$} \\
\hline & & & & & & & $\begin{array}{l}\text { Between kent portal } \\
\text { and shaft (Zone A) }\end{array}$ & $\begin{array}{l}\text { Midpoint of tunnel } \\
\text { (Between Zones B \& C) }\end{array}$ & $\begin{array}{l}\text { Between essex shaft } \\
\text { and portal (Zone D) }\end{array}$ \\
\hline \multirow[t]{5}{*}{ West Tunnel } & Zone A & $\underline{O N}$ & $\underline{O N}$ & $\underline{O N}$ & OFF & OFF & $\underline{9}$ & 5.1 & 1.2 \\
\hline & Zone B & $\overline{\mathrm{ON}}$ & $\overline{\text { OFF }}$ & $\overline{\mathrm{ON}}$ & OFF & OFF & $\overline{4} .5$ & 5.9 & 2.0 \\
\hline & Zone C & $\underline{O N}$ & OFF & $\underline{O N}$ & $\underline{O N}$ & OFF & 3.5 & $\underline{5.9}$ & 3.3 \\
\hline & Zone D & $\overline{O N}$ & OFF & $\overline{\text { OFF }}$ & $\overline{Q N}$ & $Q N$ & 2.2 & $\overline{5.4}$ & 5.1 \\
\hline & JF only & $\underline{O N}$ & OFF & OFF & OFF & OFF & 4.2 & 5.6 & 3.6 \\
\hline \multirow[t]{5}{*}{ East Tunnel } & Zone A & $\underline{O N}$ & $\underline{O N}$ & $\underline{O N}$ & OFF & OFF & $\underline{5}$ & 2.2 & 5.2 \\
\hline & Zone B & $\overline{\mathrm{ON}}$ & $\overline{\text { OFF }}$ & $\overline{O N}$ & OFF & OFF & $\overline{5}$ & 4.1 & 1.4 \\
\hline & Zone C & $\underline{O N}$ & OFF & $\underline{O N}$ & $\underline{O N}$ & OFF & 3.7 & $\underline{4.1}$ & 3.0 \\
\hline & Zone D & $\overline{\mathrm{ON}}$ & OFF & $\overline{O F F}$ & $\overline{O N}$ & $Q N$ & 1.5 & 2.9 & 5.7 \\
\hline & JF only & $\underline{O N}$ & OFF & OFF & OFF & OFF & 4.7 & 3.2 & 4.7 \\
\hline
\end{tabular}

observed for the East tunnel prediction under some ventilation scenarios. The differences are due to changes in the weather (i.e. strong wind at the portals) during the on-site measurements.

The simplicity of the model and its robustness allows the simulation of many different ventilation scenarios, as well as the effect of different fan combinations and their interaction with the extract and supply fans. The effect of wind or other external boundary conditions (e.g. difference between static pressures at the adits) can also be easily taken into account, as can the influence of the vertical shafts, stack effect, dampers or any obstacles within the tunnel. The model can also be used to calculate the distribution of pollutants or the influence of traffic flow on the average air velocity within the tunnel, as well as to make real time predictions of ventilation flows for control purposes.

The model was used to analyse the flows resulting from each of the existing ventilation configurations, related to the strategies for each of the four zones in both of the tunnels. The results are summarised in Table 1.

Studies of the ventilation required to control smoke from fires in tunnels (Kunsch, 2002; Oka and Atkinson, 1995) suggest that the critical velocity is generally of the order of $2.5-3 \mathrm{~m} / \mathrm{s}$. Thus, for Dartford, all four ventilation strategies for both tunnels should provide more than adequate smoke control in an emergency. This was previously demonstrated using CFD simulations of the entirety of both tunnels. Indeed, in both tunnels, airflows above $3 \mathrm{~m} / \mathrm{s}$ ca be achieved throughout the entire tunnel using only jet fans with no axial supply or extraction fans. Thus, the current strategies are suitable for smoke control in all considered scenarios.

Further analysis of the simulations for the East tunnel showed that some of the airflow generated by the jet fans between the Kent portal and the extract shaft is 'lost' up the extract shaft as this short shaft poses a smaller resistance to the airflow than the main portion of the tunnel does. For example, in the 'jet fans only' case, the flow in Zone $A$ is $4.7 \mathrm{~m} / \mathrm{s}$, while the flow in Zone B is only $3.2 \mathrm{~m} / \mathrm{s}$, some of the air is lost. Similarly for the fans between the Essex shaft and the portal, some of the air moved by the fans is drawn in through the Essex extract shaft as this poses a smaller resistance than the main portion of the tunnel does. This effect is further enhanced in the Zone C strategy as the central section of the tunnel effectively has its resistance increased due to the influence of the supply fan.

Using the model it is possible to demonstrate, for example, that if dampers were fitted on the extract shafts, effectively blocking the losses, the resulting flow using all jet fans would be $3.7 \mathrm{~m} / \mathrm{s}$ throughout the tunnel.

\section{Assessment of the redundancy}

One of the advantages of using the multi-scale model is that it is comparatively easy and quick to assess the consequences of making small changes to the fan configuration, thus it is possible to assess the consequences of removing individual jet fans (or pairs) from a given scenario.

For example, Fig. 10 shows the effects of varying the number of active jet fans in the Zone $C$ ventilation strategy for the West tunnel. If it is assumed that an airflow of at least $3 \mathrm{~m} / \mathrm{s}$ is required throughout Zone $\mathrm{C}$ in this incident scenario, then it can be clearly seen that more than two pairs of jet fans are required to provide this magnitude of flow.

Similar calculations for the other zones reveal that, to generate a flow of at least $3 \mathrm{~m} / \mathrm{s}$ in each of the incident zones, a minimum of three jet fan pairs are required in the Zone $B$ scenario, only two pairs are required in the Zone $D$ scenario and no jet fans are required in the Zone A scenario; in this instance sufficient flow can be generated by the axial extract fans on their own.

Thus it is clear that several pairs of jet fans in the West tunnel may be safely taken out of use for maintenance or refurbishment, whilst still maintaining sufficient flow control capabilities for any of the considered incident scenarios.

In the East tunnel the situation is more complex due to the positioning of the fans between the portals and the shafts. An example of the results for the Zone C strategy is shown in Fig. 11. Here it is generally found that the majority of jet fans are required to produce 
F. Colella, G. Rein, R. Carvel, P. Reszka, J.L. Torero, (2010) Analysis of the ventilation systems in the Dartford tunnels using a multi-scale modelling approach, Tunnelling and Underground Space Technology 25, pp. 423-432. Open access version at http://www.era.lib.ed.ac.uk/handle/1842/1152

the required level of flow in the central section of the tunnel.

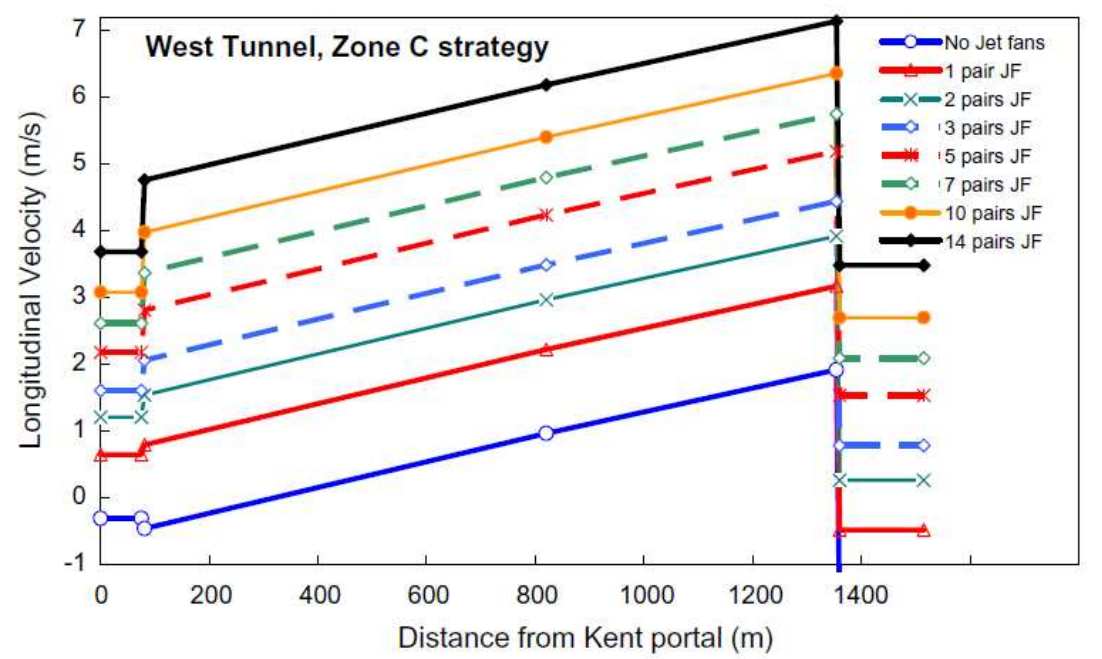

Fig. 10. Results for the West Tunnel, using the strategy for Zone $C$ (Kent supply on, Essex extract on), varying the number of active jet fan pairs. (Note: Zone C extends from approximately $700 \mathrm{~m}$ into the tunnel to $1370 \mathrm{~m}$ )

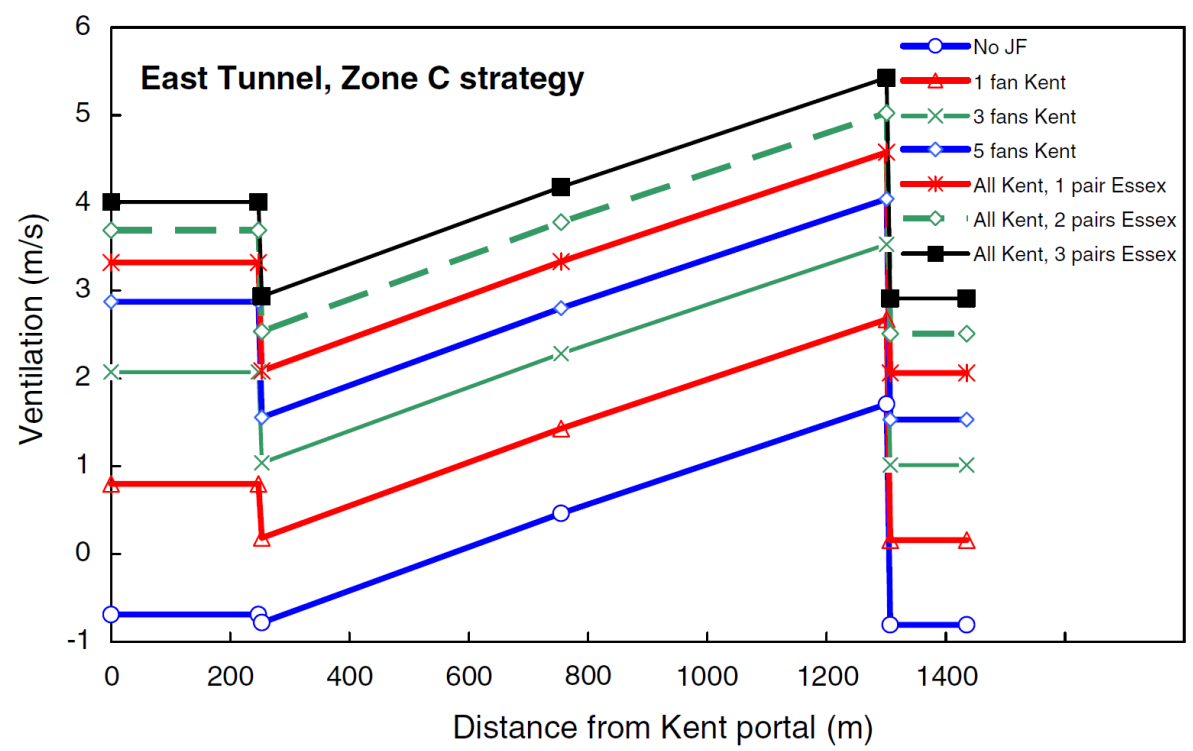

Fig. 11. Results for the East Tunnel, using the strategy for Zone C (Kent supply on, Essex extract on), varying the number of active jet fans. (Note: Zone C extends from approximately $700 \mathrm{~m}$ into the tunnel to about $1300 \mathrm{~m}$ )

A. In the Zone A ventilation strategy, only one pair of jet fans on the Essex incline are required to produce a longitudinal flow of 3 $\mathrm{m} / \mathrm{s}$ in Zone A.

B. In the Zone B ventilation strategy, all three pairs of jet fans on the Essex incline (or four Kent fans and one pair at Essex) are required to produce a longitudinal flow of 3 $\mathrm{m} / \mathrm{s}$ in Zone B.

C. In the Zone $\mathrm{C}$ ventilation strategy, at least four Kent jet fans plus one pair of Essex jet fans are required to produce a longitudinal flow of $3 \mathrm{~m} / \mathrm{s}$ in Zone C. It is also observed that the Zone B strategy (no supply fans) results in a higher flow in Zone $\mathrm{C}$ than the Zone C strategy does.
D. In the Zone $D$ ventilation strategy, at least three jet fans on the Kent incline are required to produce a longitudinal flow of $3 \mathrm{~m} / \mathrm{s}$ in Zone D.

Thus, while there is some redundancy in the East tunnel ventilation system, there is considerably less redundancy than in the West tunnel. However, it appears that one or two fans may be safely taken out of service in the East tunnel at any given time for maintenance purposes.

\section{Conclusions}

The ventilation systems in the Dartford tunnels have been analysed using a multi-scale modelling 
F. Colella, G. Rein, R. Carvel, P. Reszka, J.L. Torero, (2010) Analysis of the ventilation systems in the Dartford tunnels using a multi-scale modelling approach, Tunnelling and Underground Space Technology 25, pp. 423-432. Open access version at http://www.era.lib.ed.ac.uk/handle/1842/1152

technique. It has been demonstrated that the systems in both tunnels are able to provide the required level of ventilation flow in each of the considered emergency ventilation scenarios, even with some fans disabled.

According to the cold-flow steady state calculations, there is a high level of redundancy in the West tunnel ventilation configuration. The system could operate at the desired levels of ventilation even if several pairs of jet fans were disabled. There is less redundancy in the East tunnel system, but the system could still provide sufficient ventilation capacity if one or two fans were disabled.

The multi-scale model has been demonstrated to be accurate when compared to on-site airflow measurements and of great value for analysis of real tunnel scenarios. Analysis of many ventilation configurations can be performed in a considerably smaller timescale than with full CFD analysis. These results indicate that multi-scale modelling is an excellent methodology for simulating tunnel ventilation flows that can be extended to deal also with fire scenarios (Colella et al., 2010).

\section{Acknowledgements}

The authors would like to thank Le Crossing Company Ltd., Jacobs (UK) Ltd. and the Highways Agency for allowing access to the tunnels, assistance with the on-site measurements and permitting publication of this paper. Thanks also to Thomas Steinhaus, Kate Anderson, Nicolas Bal, Hubert Biteau, Adam Cowlard, Christian Maluk and JeanBaptiste Richon for several nights spent in cold tunnels. Special thanks to Stuart Lowe of Jacobs (UK) Ltd. for all his help with the on-site measurements.

\section{References}

Cheng, L.H., Ueng, T.H., Liu, C.W., 2001. Simulation of ventilation and fire in the underground facilities. Fire Safety Journal 36 (6), 597-619.

Colella, F., Rein, G., Borchiellini, R., Carvel, R., Torero, J.L., Verda, V., 2009. Calculation and design of tunnel ventilation systems using a twoscale modelling approach. Building and Environment 44, 2357-2367. doi:10.1016/j.buildenv.2009.03.020.

Colella, F., Rein, G., Borchiellini, R., Torero, J.L., 2010. A novel multiscale methodology for simulating tunnel ventilation flows during fires. Fire Technology, doi:10.1007/s10694-010-0144-2.

Ferro, V., Borchiellini, R., Giaretto, V., 1991. Description and application of tunnel simulation model. In: Proceedings of Aerodynamics and
Ventilation of Vehicle Tunnels Conference, pp. 487-512.

Fluent Inc., 2005. FLUENT 6.2. User's Guide.

Fox, R.W., McDonald, A.T., 1995. Introduction to Fluid Mechanics, fourth ed. John Wiley and Sons, Singapore.

Jacques, E., 1991. Numerical simulation of complex road tunnels. In: Proceedings of Aerodynamics and Ventilation of Vehicle Tunnels Conference, pp. 467-486.

Jang, H., Chen, F., 2002. On the determination of the aerodynamic coefficients of highway tunnels. Journal of Wind Engineering and Industrial Aerodynamics 89 8), 869-896.

Karki, K.C., Patankar, S.V., 2000. CFD model for jet fan ventilation systems. In: 10th International Symposium on Aerodynamics and Ventilation of Vehicle Tunnels. Boston, USA.

Kunsch, J.P., 2002. Simple model for control of fire gases in a ventilated tunnel. Fire Safety Journal 37 (1), 67-81.

Oka, Y., Atkinson, G.T., 1995. Control of smoke flow in tunnel fires. Fire Safety Journal 25 (4), 305322.

Riess, I., Bettelini, M., Brandt, R., 2000. SPRINT - a design tool for fire ventilation. In: Proceedings of Aerodynamics and Ventilation of Vehicle Tunnels

Conference.

Vega, M.G. et al., 2008. Numerical 3D simulation of a longitudinal ventilation system: memorial tunnel case. Tunnelling and Underground Space Technology 23 (5), 539-551.

Versteeg, H.K., Malalasekera, W., 2007. An Introduction to Computational Fluid Dynamics, second ed.. The Finite Volume Method Pearson Prentice Hall, Glasgow. 